\title{
ADDITIVE AND MULTIPLICATIVE LOOPS OF PLANAR TERNARY RINGS
}

\author{
D. R. HUGHES
}

1. Introduction. Given an arbitrary countably infinite loop $G$, does there exist a planar ternary ring whose additive or multiplicative loop is isomorphic to $G$ ? The author is grateful to Professor Trevor Evans for pointing out this question, and is prepared to answer it in the affirmative. In fact, either the additive loop, the multiplicative loop, or both can be arbitrary; furthermore, if $G$ is any countably infinite group, then there is a linear planar ring whose additive loop is isomorphic to $G$.

2. Addition and multiplication arbitrary. If $R$ is a nonempty set with distinct elements 0 (zero) and 1 (one, or the identity), and $F$ is a ternary operation such that $F(a, b, c)$ is in $R$ for all $a, b, c \in R$, and if $(\mathrm{A})-(\mathrm{E})$ below are satisfied, then $(R, F)$ is called a planar ternary ring (see $[1,2]$ ).

(A) $F(a, 0, c)=F(0, b, c)=c$, for all $a, b, c \in R$.

(B) $F(a, 1,0)=F(1, a, 0)=a$, for all $a \in R$.

(C) If $a, b, c, d \in R, a \neq c$, then there is a unique $x \in R$ for which $F(x, a, b)=F(x, c, d)$.

(D) If $a, b, c \in R$, then there is a unique $x \in R$ for which $F(a, b, x)$ $=c$.

(E) If $a, b, c, d \in R, a \neq c$, then there is a unique (ordered) pair $x, y \in R$ for which $F(a, x, y)=b, F(c, x, y)=d$.

We define addition in $R$ by $a+b=F(1, a, b)$, and multiplication by $a b=F(a, b, 0)$. Then the set $R$, under addition, forms a loop with "identity" 0 , and the set $R^{*}$ of nonzero elements of $R$, under multiplication, forms a loop with identity 1 . (Sometimes $a+b$ is defined as $F(a, 1, b)$, as in [1]; the following results carry through as well in this case.) These loops are called the additive and multiplicative loops, respectively.

Now suppose $R$ is (without loss of generality) the set of nonnegative integers, and an additive loop is defined on $R$, with "identity" 0 ; suppose also that a multiplicative loop is defined on the set $R^{*}$ of positive integers, with identity 1 . We wish to define a ternary operation $F$ on $R$ such that $F(a, b, 0)=a b$ if $a, b \in R^{*}, F(1, a, b)=a+b$ for all $a$, $b \in R$, and such that (A)-(E) are satisfied.

Received by the editors February 11, 1955. 
Let us define $F(a, 0, c)=F(0, b, c)=c$, for all $a, b, c \in R ; F(1, a, b)$ $=a+b$, for all $a, b \in R ; F(a, b, 0)=a b$, for all $a, b \in R^{*}$. Then it is easy to see that (A) and (B) are satisfied and, in fact, cannot be contradicted no matter how $F$ is defined for the remaining triples; furthermore $(\mathrm{C})-(\mathrm{E})$ are not contradicted.

Our work is simplified by the following:

LEMMA 1. In the axioms of a planar ternary ring, (E) can be replaced by $\left(\mathrm{E}^{\prime}\right)$.

( $\left.\mathrm{E}^{\prime}\right)$ If $a, b, c, d \in R, a \neq c$, then there is at least one pair $x, y \in R$ for which $F(a, x, y)=b, F(c, x, y)=d$.

Proof. Suppose $F(a, x, y)=b=F(a, u, v), F(c, x, y)=d=F(c, u, v)$, where $a \neq c$. If $x=u, y \neq v$, then $F(a, x, y)=b=F(a, x, v)$, which contradicts (D); so we assume $x \neq u$. But then the equation $F(z, x, y)$ $=F(z, u, v), x \neq u$, has the two distinct solutions $z=a, z=c$, which contradicts (C). So $x=u, y=v$, and we are done.

Hence in order to define our planar ternary ring, it is sufficient to assure that all equations of type (C) and (D) have unique solutions, and that all equations of type (E) have at least one solution. Let us well-order all 4-tuples $(a, b, c, d), a \neq c$, and all 3-tuples $(a, b, c)$, where $a, b, c, d \in R$. Then we shall proceed through the ordering, "solving" equations as we go.

At any point in the process, note that if $a \neq 0,1, b \neq 0, c \neq 0$, then $F(a, b, c)$ is defined for only finitely many $a, b, c$.

I. Equations of type (C). Suppose $(a, b, c, d), a \neq c$, is the first 4-tuple for which $F(x, a, b)=F(x, c, d)$ has no solution $x$.

CASE 1. Suppose $a, b, c, d$ all nonzero. Let $n$ be any element for which $F(n, a, b)$ and $F(n, c, d)$ are not yet defined. If $F(n, a, b)=p$ $=F(n, c, d)$ contradicts $(\mathrm{C})$ or $(\mathrm{D})$, then at least one of the following holds:

$$
\begin{array}{cr}
p=F(n, u, v), F(k, a, b)=F(k, u, v), & u \neq a, k \neq n . \\
p=F(n, u, v), F(k, c, d)=F(k, u, v), & u \neq c, k \neq n . \\
F(n, a, g)=p, & g \neq b . \\
F(n, c, g)=p, & g \neq d .
\end{array}
$$

Consider (1). Unless $u=0$ or $v=0$, there are only finitely many $u, v$ for which $F(n, u, v)$ is defined, whence (1) is possible for only finitely many $p$. If $u=0$, then $F(k, a, b)=p$, but $F(k, a, b)$ is defined for only finitely many $k$, so again (1) is satisfied for only finitely many $p$. If $v=0$, then $p=n u, F(k, a, b)=k u$. If $k \neq 0$, then this is possible for only finitely many $k$, hence for finitely many $u$, hence for finitely many $p$. 
If $k=0$, then (1) implies $b=0$, which is contradictory. So (1), and similarly (2), are possible for only finitely many $p$.

Clearly (3) and (4) are possible for only finitely many $p$. So we can, by avoiding a finite set of values for $p$, define $F(n, a, b)$ $=F(n, c, d)=p$.

CASE 2. If $a=0$, then we wish to satisfy the equation $F(x, c, d)=b$. Suppose $n$ is an element for which $F(n, c, d)$ is not defined. (Note that $c \neq 0, d \neq 0, b$.) Then $F(n, c, d)=b$ leads to a contradiction only if one of the following holds:

$$
\begin{gathered}
b=F(n, u, v), F(k, c, d)=F(k, u, v), \quad u \neq c, k \neq n . \\
F(n, c, g)=b, \\
g
\end{gathered}
$$

Consider (5). Unless $u=0$ or $v=0, F(n, u, v)$ is defined for only finitely many $n$, hence (5) holds for only finitely many $n$. If $u=0$, then (5) becomes $F(k, c, d)=b$, which contradicts our assumption that $F(x, c, d)=b$ has no solution. If $v=0$, then (5) becomes $b=n u$, $F(k, c, d)=k u$. If $k \neq 0$, then this defines a finite set of elements $u$, hence a finite set of elements $n$; if $k=0$, then (5) implies $d=0$, which is contradictory. So, in all cases, (5) is satisfied for only finitely many $n$.

Clearly (6) is satisfied for only finitely many $n$.

CASE 3. If $b=0$, then we wish to satisfy $x a=F(x, c, d)$; note that $c \neq 0, d \neq 0$. If $n$ is an element such that $F(n, c, d)$ is not defined yet, and if $F(n, c, d)=n a$, then we are led to a contradiction only if one of the following holds:

$$
\begin{array}{rlr}
n a=F(n, u, v), F(k, c, d)=F(k, u, v), & u \neq c, k & \neq n . \\
F(n, c, g)=n a, & g & \neq d .
\end{array}
$$

Consider (7). As before, $F(n, u, v)$ is defined for only finitely many $n$, unless $u=0$ or $v=0$. If $u=0$, then (7) becomes $F(k, c, d)=n a$, which is possible for only finitely many $n$, since $F(k, c, d)$ is defined for only finitely many $k$. If $v=0$, then $n a=n u$, or $a=u$, and $F(k, c, d)$ $=k a$, which contradicts our assumption that $F(x, c, d)=x a$ has no solution. So (7) is always satisfied for only finitely many $n$.

Clearly (8) is possible for only finitely many $n$.

So, in all cases, an equation of type $(\mathrm{C})$ can always be solved in a "non-contradictory" fashion.

II. Equations of type (E). Suppose $(a, b, c, d), a \neq c$, is the first 4-tuple for which $F(a, x, y)=b, F(c, x, y)=d$ has no solution.

CASE 1. Suppose $a \neq 0,1, c \neq 0,1$. Suppose $m$ and $n$ are elements such that $F(a, m, n), F(c, m, n)$ are not yet defined. Then $F(a, m, n)$ $=b, F(c, m, n)=d$ leads to a contradiction only if one of the following holds: 


$$
\begin{array}{cr}
b=F(a, u, v), F(p, m, n)=F(p, u, v), & u \neq m, p \neq a . \\
d=F(c, u, v), F(p, m, n)=F(p, u, v), & u \neq m, p \neq c . \\
F(a, m, g)=b, & g \neq n . \\
F(c, m, g)=d, & g \neq n .
\end{array}
$$

Consider (1). $F(a, u, v)=b$ holds for only finitely many $u, v$, and for all but finitely many $m, n, F(p, m, n)$ is defined only if $p=0$ or 1 . But then $n=v$ (if $p=0$ ) and $m+n=u+v$ (if $p=1$ ) is possible for only finitely many $m, n$. So (1), and similarly (2), are possible only for finitely many $m, n$. (Note that neither $m$ nor $n$ is zero.)

Clearly (3) and (4) are only possible for finitely many $m$.

CASE 2. Suppose $a=0$. Then $c \neq 0,1$. If $F(c, n, b)$ is not yet defined, then $F(c, n, b)=d$ leads to a contradiction only if one of the following occurs:

$$
\begin{array}{cr}
d=F(c, u, v), F(k, n, b)=F(k, u, v), & u \neq n, k \neq c . \\
F(c, n, g)=d, & g \neq b .
\end{array}
$$

Consider (5). If $u=0$, then this becomes $F(k, n, b)=d$. If $k=0$, then $b=d$, and $F(c, 0, b)=d$, contrary to our assumption that $F(c, x, b)$ has no solution. If $k=1$, then $n+b=d$, which is possible for exactly one $n$. If $k \neq 0,1$, then $F(k, n, b)=d$ holds for finitely many $k$, unless $b=0$; but $b=0$ implies that $F(c, x, b)=c x=d$ already has a solution. So $F(k, n, b)=d$ holds for only finitely many $n$.

If $v=0$, then $d=c u, F(k, n, b)=k u$. If $k=0$, then $b=0$, and again $F(c, x, b)=d$ already has a solution. If $k=1$, then $n+b=u, d=c u$, and this defines exactly one $n$. If $k \neq 0,1$, then $c=d u$ defines exactly one $u$, and $F(k, n, b)=k u$ defines finitely many $k$ and $n$ (unless $b=0$, which is impossible).

If $u \neq 0, v \neq 0$, then $d=F(c, u, v)$ holds for at most finitely many $u$, $v$, and $F(k, u, v)$ is defined for at most finitely many $k$. So $F(k, n, b)$ $=F(k, u, v)$ holds for only finitely many $n$.

Clearly (6) holds for at most finitely many $n$.

CASE 3. Suppose $a=1$, whence $c \neq 0,1$. Then we wish to solve $x+y=b, F(c, x, y)=d$. Note that $b \neq d$, for otherwise $x=0, y=b$ would be a solution. If $m$ and $n$ are elements such that $m+n=b$, and $F(c, m, n)$ is not defined (there must be such elements, since there are infinitely many $m$ and $n$ for which $m+n=b)$, then $F(c, m, n)=d$ leads to a contradiction only if one of the following holds:

$$
\begin{array}{rr}
d=F(c, u, v), F(k, m, n)=F(k, u, v), & k \neq c, u \neq m . \\
F(c, m, g)=d, & g \neq n .
\end{array}
$$


Consider (7). If $u=0$, then $F(k, m, n)=d$; but $F(1, m, n)=b$, $F(k, m, n)=d$ is possible for only one pair $m, n$, and $F(k, m, n)$ is defined for only finitely many $k$, unless $k=1$. But $k=1$ implies $m+n$ $=d=b$, which is contradictory.

If $v=0$, then $d=c u, F(k, m, n)=k u$. If $k=0$, then $n=0, m=b$, and $F(c, b, 0)=d$, which contradicts our assumption that $F(c, x, y)=d$, $x+y=b$ has no solution. If $k=1$, then $m+n=u=b$, so $d=c b$; hence $F(1, b, 0)=b, F(c, b, 0)=d$, which is contradictory. If $k \neq 0,1$, then $d=c u$ defines one $u$, and $F(k, m, n)=k u$ defines finitely many $k$, hence finitely many $m$ (for $F(k, m, n)$ is defined for only finitely many $k$, $m, n)$.

If $u \neq 0, v \neq 0$, then $d=F(c, u, v)$ is possible for at most finitely many $u, v$. Hence $F(k, u, v)=F(k, m, n)$ is possible for at most finitely many $m, n$ (where we recall that $m+n=1$ ).

Clearly (8) is possible for only finitely many $m, n$.

So we can always solve an equation system of type (E) in a "noncontradictory" fashion.

III. Equations of type (D). Suppose $(a, b, c)$ is the first 3-tuple for which $F(a, b, x)=c$ has no solution. Note that $a \neq 0,1, b \neq 0, a b \neq c$. If $n$ is an element such that $F(a, b, n)$ is not defined, then $F(a, b, n)=c$ is contradictory only if:

$$
c=F(a, u, v), F(p, b, n)=F(p, u, v), \quad p \neq a, u \neq b .
$$

If $u=0$, then $F(p, b, n)=c$. If $p=0$, then we must have $n=c$; if $p=1$, then we have $b+n=c$. If $p \neq 0,1$, then $F(p, b, n)=c$ is possible for only finitely many $p$ and $n$.

If $v=0$, then $c=a u, F(p, b, n)=p u$, and as above, this defines at most finitely many $n$.

If $u \neq 0, v \neq 0$, then $F(a, u, v)$ is possible for only finitely many $u, v$. If $p=0$, then $n=v$, and if $p=1$, then $b+n=u+v$. If $p \neq 0,1$, then $F(p, b, n)=F(p, u, v)$ holds for at most finitely many $p$ and $n$.

So only finitely many $n$ satisfy (1), so we can solve an equation of type (D) in a "noncontradictory" fashion.

IV. Remaining equations. If $(a, b, c)$ is the first 3-tuple such that $F(a, b, c)$ is not defined, then note that $a \neq 0,1, b \neq 0, c \neq 0 . F(a, b, c)$ $=n$ is contradictory only if one of the following holds:

$$
\begin{aligned}
n=F(a, u, v), F(k, b, c)=F(k, u, v), \quad k \neq a, u & \neq b . \\
F(a, b, g)=n, & g \neq c .
\end{aligned}
$$

Consider (1). If $u=0$, then $F(k, b, c)=n$, which is possible for at most finitely many $n$. If $v=0$, then $n=a u, F(k, b, c)=k u$; if $k=0$, then $c=0$, which is contradictory; if $k=1$, then $b+c=u, a u=n$, which is 
possible for only one $n$. If $k \neq 0,1$, then $F(k, b, c)=k u$ is possible for only finitely many $k$ and $u$, hence $n=a u$ is possible for only finitely many $n$.

If $u \neq 0, v \neq 0$, then $F(a, u, v)$ is defined for only finitely many $u, v$, so $F(a, u, v)=n$ is possible for only finitely many $n$.

Clearly (2) holds for at most finitely many $n$, so we can define $F(a, b, c)$ in a "noncontradictory" fashion.

Thus, by repeating the cycle of these four steps, it is clear that we can define $(R, F)$ to be a planar ternary ring. It is fairly obvious that either the additive or the multiplicative loop could have been omitted in the construction.

3. Associative addition. Suppose $R$ is a countable group under the operation ( + ), with "identity" 0 (zero). We wish to define a planar ternary ring $(R, F)$ whose additive loop is given by $(+)$, and which is linear; i.e., $F(a, b, c)=a b+c$, for all $a, b, c \in R$. Note that this demands Hall's Theorem $L$ in a special sense in the projective plane coordinatized by $(R, F)$.

Let 1 be some fixed nonzero element of $R$, and define $0 a=a 0=0$, all $a \in R$, and $1 a=a 1=a$, all $a \in R$. Since $(R, F)$ is to be linear with associative addition, the equations of type $(C)$ can be replaced by equations $x a=x b+c, a \neq b$; the equations of type (E) can be replaced by $a x=b+c x, a \neq c$, and the equations of type (D) are automatically satisfied.

Suppose $x a=x b+c, a \neq b$, has no solution; note that then $a, b$, and $c$ must be nonzero.

If $a=1$, and $n$ is an element such that $n b$ is not yet defined, then $n b=n-c$ is contradictory only if:

$$
n-c=n u+v, k b=k u+v, \quad u \neq b, k \neq n .
$$

If $u=1$, then $v=-c$, and $k b+c=k$, which contradicts our assumption that $x=x b+c$ has no solution. If $u=0$, then $n-c=v, n=k b+c$; but this holds for only finitely many $n$. If $u \neq 0,1$, then (1) becomes (by subtraction) $n=n u-k u+k b+c$; since $n u$ is defined for only finitely many $n$, this is possible for only finitely many $n$.

If $a \neq 1, c \neq 1$, and $n$ is an element such that $n a$ and $n b$ are not yet defined, then $n a=n b+c=p$ (for a fixed $n$ ) is contradictory only if one of the following holds:

$$
\begin{aligned}
p & =n u+v, k a=k u+v, & & k \neq n, a \neq u . \\
p-c & =n u+v, k b=k u+v, & & k \neq n, b \neq u .
\end{aligned}
$$

Eliminating $v$ in each pair, we therefore demand: 


$$
\begin{array}{ll}
p \neq n u-k u+k a, & \text { if } k \neq n, a \neq u . \\
p \neq n u-k u+k a+c, & \text { if } k \neq n, b \neq u .
\end{array}
$$

Consider (4). For a fixed $n, n u$ is defined for only finitely many $u$, and $k a$ is defined for only finitely many $k$, so (4) holds for all but finitely many $p$. Similarly for (5).

Now suppose $a x=b+c x, a \neq c$, has no solution; as before, then, $a, b$, and $c$ are all nonzero.

If $a=1$, and $n$ is an element such that $c n$ is not yet defined, then $c n=-b+n$ leads to a contradiction only if:

$$
-b+n=c u+v, k n=k u+v, \quad k \neq c, u \neq n .
$$

If $k=1$, then (6) implies $u=b+c u$, which contradicts our assumption that $x=b+c x$ has no solution. If $k=0$, then $v=0, n=b+c u$, but this is possible for only finitely many $n$, since $c \neq 1$. If $k \neq 0,1$, then (6) becomes $n=b+c u-k u+k n$; since $k n$ is defined for only finitely many $n$, this is possible for only finitely many $n$.

If $a \neq 1, c \neq 1$, and $n$ is an element such that $a n, c n$ are not yet defined, then $a n=b+c n=p$ (for a fixed $n$ ) is contradictory only if one of the following holds:

$$
\begin{aligned}
p & =a u+v, k n=k u+v, & & k \neq a, u \neq n . \\
-b+p & =c u+v, k n=k u+v, & & k \neq c, u \neq n .
\end{aligned}
$$

These can be handled exactly like our treatment, of (4) above.

Finally, if $a b$ is not yet defined (whence certainly $a \neq 0,1, b \neq 0,1$ ), then $a b=p$ is contradictory only if:

$$
p=a u+v, k b=k u+v, \quad k \neq a, u \neq b .
$$

Again, this can be treated like (4).

Hence by a cyclic repetition of the above three steps, proceeding through a well-ordering of 3 -tuples and 2-tuples, we can define multiplication such that the ternary ring $(R, F)$ given by $F(a, b, c)$ $=a b+c$ is planar.

The author does not know whether an arbitrary countable loop can be the additive loop of a linear planar ring; since this would imply that any additive condition whatsoever (in countable loops, at least) was compatible with Theorem L, one might conjecture that it is not possible.

Added in proof. 1. The construction of a linear planar ring whose additive loop is an arbitrary (countable) group gives a solution to the following problem, which has already been solved by Pickert 
(Nichtkommutative cartesische Gruppen, Archiv der Mathematik vol. 3 (1952) pp. 335-342) in a slightly different fashion: does there exist an affine plane with a noncommutative group of translations? Using the coordinatizing scheme of [2], the mappings $\phi_{x}$ given by $\phi_{x}$ : $(a, b) \rightarrow(a, b+x), \phi_{x}:[m, k] \rightarrow[m, k+x], \phi_{x}:[\infty,(k, 0)] \rightarrow[\infty,(k, 0)]$, are all translations, and in fact, form a group isomorphic to the additive group of the planar ring.

2. The construction of a planar ternary ring with arbitrary additive loop can easily be generalized so that any finite set of mutually orthogonal latin squares of countably infinite order can be extended to a complete set of mutually orthogonal latin squares. For details of the correspondence between the latin squares and the ternary ring, see [2].

\section{BIBLIOGRAPHY}

1. M. Hall, Projective planes, Trans. Amer. Math. Soc. vol. 54 (1943) pp. 229-277.

2. D. R. Hughes, Planar division neo-rings, Trans. Amer. Math. Soc. vol. 80 (1955) pp. 502-527.

The UNIVERSITY OF WisConsin 